\title{
TECHNICAL RESERVES IN NON-LIFE INSURANCE WITH PARTICULAR REFERENCE TO MOTOR INSURANCE
}

\author{
R. E. BEARD \\ London
}

\section{Background}

At the ASTIN Colloquium held in Lucerne I reported on the request from the 6th Conference of European Insurance Supervisors for co-operation in the studies being made into the subject of technical reserves in non-life insurance. Copies of notes prepared for the 6th Conference were distributed to members and an advisory committee was formed to assist in dealing with the request. Two reports have been sent to all members of this committee. It has not been practicable to prepare further reports and the present paper sets out the current position of the work being done.

2. It will be recalled that in a number of papers I have represented that research into methods of determining technical reserves has been severely restricted because of the absence of suitable statistics on which to experiment. This point has been well taken by the European Insurance Supervisors and recently suitable statistics of motor insurance on which to experiment have been made available on a confidential basis relating to 6 Swedish, 7 German, 5 French, 6 Italian and 4 British companies. These returns, whilst not compiled on a uniform basis, provide an immense amount of data not previously obtainable, and it will be appreciated that the analysis of the data is a task of considerable magnitude.

3. I have made certain preliminary studies as a result of which a number of points of detail have arisen which have been referred back for clarification or explanation. Nevertheless these studies have provided a very useful background to the whole problem and the present paper is written against this background and of my earlier papers on the topic. Since the detailed statistics are confidential and agreement for publication would mean some delay, only the broadest indications of numerical values are given. Nevertheless, 
the comments made are based on the studies of the figures for the 28 companies and thus have a considerable practical utility.

4. Before entering on the details of the paper I must express thanks to the 6th Conference of European Insurance Supervisors for their help in providing the statistics and to the O.E.C.D. who are engaged on parallel studies. I was invited to a meeting of a working party of O.E.C.D. to talk about the actuarial approach and also to the recent meetings of the 6th Conference to report progress on the analysis of the statistics.

\section{Basis of current approach}

5. Since the reserves required are essentially an estimate of the future outgo in respect of commitments entered into prior to the end of the accounting period in question, it is necessary to find some method of using recorded facts to provide a basis of forward estimating. This means that rational explanations must be found for variations shown in the facts so that some basis exists for carrying calculations into the future. Furthermore, since there will be a variation in the emergence of the future results as compared with the estimates from random fluctuations, it must also be possible to make a reasonable estimate of the distribution of such fluctuations so that an appropriate margin can be determined. It is hardly sufficient as a commercial rule to merely specify a large margin as this could result in undue restriction on the development of smaller (or specialist) companies and also require unnecessarily large capital reserves to be tied up in the business.

6. As a basic principle on which to build calculations it is assumed that for a homogeneous portfolio in stable conditions the claim frequency rate (i.e. number of claims per Ioo cases exposed) will be constant apart from random fluctuations and the distribution of claims by amount will be stable apart from random fluctuations. It is hardly possible to produce direct observations to prove the validity of these two basic assumptions because of the difficulty of establishing experimental conditions in which the factors affecting the frequency and mean claim remain steady, but all the evidence available points to their truth. It may be observed that were they in fact not true, there could be no rational basis for the calculation of insurance premiums. 
7. To build on this principle it is necessary to study the variations in practical conditions of these quantities and to identify those factors which give rise to non-random variations. When the nonrandom variations have been identified, estimates of future results can be made and also the significance of random variations determined.

\section{Technical reserves required}

8. The technical reserves required can be classified as follows:

(a) Reserves for unexpired risks

(b) Reserves for incurred but unreported claims

(c) Reserves for outstanding claims

(d) Fluctuation reserves

In the following paper the claim component only is first considered. The requirements for (a) and (b) are determined by finding the expected numbers of claims and multiplying these numbers by an appropriate average claim. The reserve (c) is found by multiplying the number of incurred claims in each year by an appropriate average and deducting therefrom the actual settlements to the close of the account concerned. This resulting outstanding is then subjected to certain other checks. The fluctuation reserve calculations are described later.

9. This approach leads to the basic summary control as follows: Year of Account 1965

$\begin{array}{lllll}\text { Year } & \begin{array}{l}\text { No. of } \\ \text { claims }\end{array} & \begin{array}{l}\text { Expected } \\ \text { Av. settlement Settlements }\end{array} & \begin{array}{l}\text { Expected } \\ \text { 31.12.65 }\end{array} \text { = Outstanding } \\ \text { I961 } & & & \\ 1962 & & & \\ 1963 & & & \\ 1964 & & & \\ 1965 & & \end{array}$
Total

Io. The numbers of claims and the payments are factual figures derived from the company records. The expected average settlement is a judgement figure derived as explained below. The number of years for which the details are required depends on the class of business. For own damage claims in motor insurance practically 
all the claims will be settled at the end of the second year and any earlier claims outstanding could be dealt with on a separate comparison basis. For motor third party claims at least 5 years will be needed in this form. Earlier years can be dealt with on a basis of comparing the company estimates at the previous year with the settlements and current outstandings to determine the evolution of the estimates.

II. Certain supplementary statements will be needed to complete the basic summary. These are needed to control the numbers of claims and the distribution of claims settlements (numbers and amounts) in time. So soon as a few years statements become available the estimation problems will become relatively straightforward. The various components of the summary are now discussed in detail against the studies of the 28 companies' figures.

\section{Numbers of claims}

I2. It is quite clear that there are a number of different ways in which the number of claims is defined and the important point is to ensure consistency. The treatment of zero claims also varies between companies, probably a reflection of different internal procedures. A major difference in definition arises when two or more claims can arise under a single policy and there was evidence in some companies of a change in definition over the period of their returns. Consistency in a given company is essential, but either method may be used, i.e.

(i) To treat all claims arising from one incident as a single claim

(ii) To treat each claim as a separate item.

I3. Method (ii) will clearly give rise to a greater number of claims for the same number of incidents as compared with method (i). There will however be a rather lower proportion of zero claims and also a lower proportion of part payments. The average claim will also be lower under method (ii). It is important that the method being used be properly defined and any changes in procedure made clear.

I4. The number of claims notified in a year can be considered as the sum of those arising from incidents in the account year and 
those arising from incidents in earlier years. In theory the numbers should be allocated to their years of incident and an adjustment made for unreported claims at the close of the period. In practice it can be questioned whether this refinement is justified in terms of the quantities involved as the accounting detail required is quite extensive. From the figures available the late reportings are of the order of a few per cent of the notified claims, the maximum being about $13 \frac{1}{2} \%$. For any given company the proportion of late reportings is fairly consistent, confirmation that the proportion is related to the organisation of the company, but in all cases the numbers were important enough to justify a special reserve (8(b)).

I5. If the number of claims is taken as the number actually notified in the account year and the late reportings are $10 \%$, then if the business is growing at the rate of $10 \%$ p.a. the number of notifications will be $1 \%$ less than the true incurred claims for the year. If the claim frequency rate is .Io then for a total of $I, 000$ expected claims the standard deviation will be rather more than $3 \%$ and it will be difficult to detect an error of $1 \%$ in the frequency rate. For larger numbers of claims the standard deviation is smaller and the error might be detectable.

16. The real problem arises from the continued and rapid growth in the motor business so that a systematic bias exists if the figures are not corrected. In practical conditions it would seem adequate if the numbers of claims were taken as the intimated claims, an estimate made of the proportion of late reportings from the company figures and adjustments made to the claim frequency rate and the average claim to compensate for the late reportings of the earlier years. These adjustments will be mentioned again later.

I7. The remaining item on the number of claims relates to the numbers of settled claims. If the numbers are taken as notifications and settlements in accounts years, the outstanding claims are obtained subject to re-opened cases. In theory these should be related back to their proper year of arising, but again it can be questioned if the clerical effort is really worth while, although there are certain dangers which must be considered. For "normal" 
companies the proportion of re-opened cases is about $\mathrm{I} \%$ of the total claims so that any error from treating them as new claims in the account year of re-opening must be small (even though it is systematic). However a situation could arise in which a company systematically closed cases as zeros, only to open them again at a future date. By this means it would be possible for a rapidly growing company to throw forward an appreciable proportion of its claims and thus underprovide for the outstandings.

I8. A practical rule is to require re-opened cases to be separately recorded. Consideration of the claim frequency rate and the proportion of zero claims with an appropriate time lag should then provide an indication if the re-opened cases are unduly high in which case further details could be sought.

I9. Reference has been made to the numbers of zero claims. The incidence of such claims can be markedly influenced by the administration of the particular company, but is also linked up with the definition of what constitutes one claim. The numbers can be recorded separately or they can be regarded as part of the distribution of claims by amounts, but since this latter information is extensive and expensive to prepare as a routine operation, the separate recording of zero cases seems the better treatment. The fact that the number of zero cases can fluctuate by administrative action also suggests that figures relating to the numbers of claims on which some payment is made will be more stable.

20. The proportion of zero claims varies quite considerably over the data examined from a minimum of a few per cent to instances where over $50 \%$ of the claims are zeros. For a given company the proportion is characteristic of that company so that once a few years figures are available it will become apparent if there is an unusual movement. Similarly the distribution of the zero claims in time is characteristic of each company, i.e. they are eliminated at about the same durations, but shifting in durations can seriously affect the average claims and for stability in comparisons they are best eliminated.

2I. From these considerations the following appears to be an economical statement relating to the numbers of claims: 
Accounting Year 1965

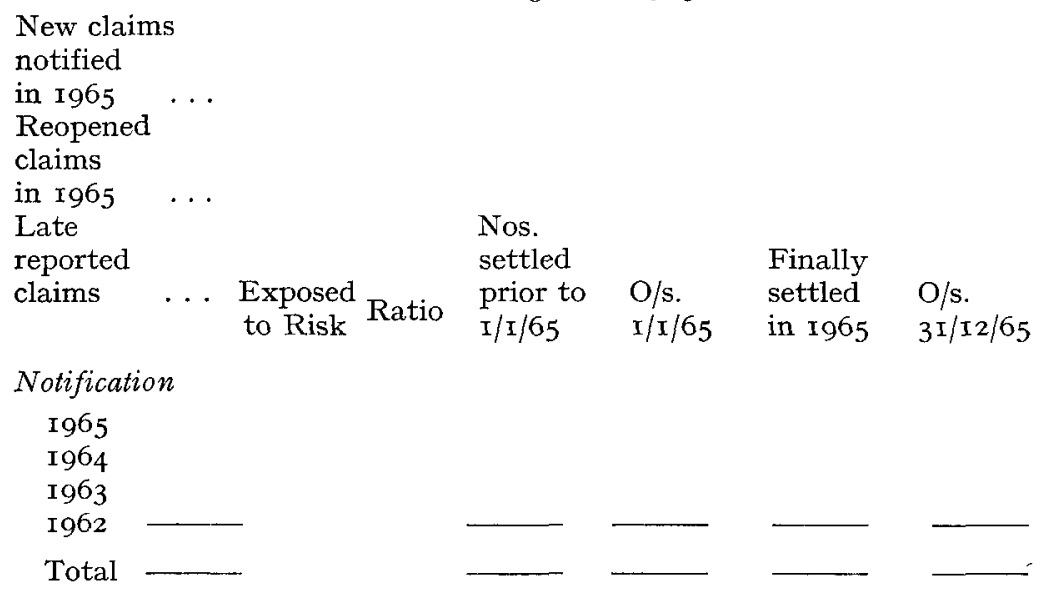

22. Assuming the vehicle years exposed to risk are available and the above details for a number of years, the following calculations can be made to determine the internal consistency of the figures:

(a) Ratio of total notifications to exposed to risk will give the claim frequency rate. Subject to later comments on the subject of "mix" this should be a relatively stable quantity. If violent fluctuations occur search can be made for reasons and identified with recognised features. In the cases examined weather conditions probably gave rise to some minor variations; change in claim definition seemed responsible for others; sudden influx of a large low frequency class (mopeds) occurred in another. A slow drift in the ratio may be due to a change in the "mix" of the business; this can be checked provided some details are provided of the composition of the account. There may be a change in the basic underlying risk, i.e. an increase in the accident level in the country concerned.

(b) The re-opened claims mainly arise from the previous year's claims and the variation in these should thus be considered relative to this figure. If there is evidence of excessive numbers an addition should be made to the late reportings to take care of the position. Special attention should be given 
to the proportion of zeros in the claims settled in the year of notification in such cases.

(c) The late reportings similarly refer to the previous year's notifications and should be compared with them.

(d) There should of course be agreement between the outstandings and the notifications less settlements.

(e) The ratio of number of settlements to the number of notifications will be an appropriate comparison figure when a few years' returns are available.

(f) The proportion outstanding should also be studied in successive years at the appropriate durations.

23. The numbers of zero claims could be included in the foregoing statement or they can be included in the statement relating to amounts of claims. The figures to be studied are the ratio of zero claims to settlements at successive durations. From the available records the normal pattern is for this ratio to increase with increase in duration, i.e. the proportion of zero claims in the first year settlements may be $20 \%$ rising to $35 \%$ for the first 5 years settlements. For any given company the pattern is consistent, although different companies will show different patterns.

\section{Amounts of Claims}

24. The basic statistic is the average settled claim and the returns have to be designed to throw up the relevant information in a suitable form. From the studies made there is every justification for the basic hypothesis that the average claim is a reasonably stable quantity provided the portfolio is homogeneous, but, as with the numbers of claims, a number of factors have been discovered which obscure the stability. Unless allowance is made for these future estimates may be on an unreliable basis.

25. The first important point to note is that the factual information is the payments made and the appropriate statistic to record is the average settled claim, i.e. the total payments divided by the numbers of claims in respect of which the payments are made. For the year I 965 we can find the average ist year settlement value for the I 965 claims, the average Ist and 2 nd year settlement for I964 claims and so on. Once a few years statements are available 
the variation in the average Ist year claim, the Ist and and year claims etc., can be studied. In all the figures examined the average first year claim showed a steady increase with time, the annual rate of increase bearing a fairly close relationship with the wages index for the respective countries. Similar results were found for the Ist and 2nd year figures and for the Ist, and and 3rd years. In general the data was for the years I960-I964 so that the later durations were not sufficient to develop a time trend.

26. The first year settlements are, of course, the smaller claims and the average settled first year claim can be as low as about one-half of the ultimate average. Since we are interested in using the figures to arrive at the ultimate average settlement, the progression of the averages at successive durations is important. Typical runs of values are as follows:

$\begin{array}{lrrrrrc} & \text { Ist } & \text { 2nd } & 3^{\text {rd }} & 4^{\text {th }} & 5^{\text {th }} & \text { ultimate } \\ \text { (a) } & 44 & 5^{6} & 7^{2} & 8 \mathrm{I} & 87 & \text { roo } \\ \text { (b) } & 7^{2} & 84 & 94 & 97 & - & \text { roo }\end{array}$

The averages have been expressed in the terms of the estimated ultimate figure which, for the particular case, has been found by using the company estimates of outstanding claims at the end of the $5^{\text {th }}$ year (a) and 4 th year (b). It may be noted that the number of claims settled after the $5^{\text {th }}$ year is very small and the difference from 87 to roo in case (a) is accounted for by a few very large settlements.

27. Clearly the problem of estimating the trend from the $5^{\text {th }}$ year to the ultimate is not easy and until data have been accumulated to map this region more closely reliance will have to be made on the company estimates. The position varies from country to country, with some showing about $5 \%$ of claims (by amounts) outstanding at the $5^{\text {th }}$ years while others show figures of $15 \%$. Nevertheless the estimation error will normally be small relative to the ultimate average since it is the error in the outstandings which, at this point in time, should be a fairly close approximation to the actual settlement.

28. From the table of average settlements it has been found practicable to arrive at a reasonable sequence of estimates for the ultimate settlement figures for successive years of notification, 
the broad basis being to use the annual increase figures from the early durations to arrive at increases for the ultimate figures. By this means the critical information for completing the schedule in para. 9 can be developed.

29. There are however a number of points which have arisen which should be noted. In the first place the increase in the average claim when changes in the "mix" of the business are eliminated is associated with the change in value of money. To use the past values in the manner suggested implies that future changes will continue as in the past. For example claims arising in I960 will be settled over later years and the settlements in say I965 will reflect monetary levels of that year. This trend will be reflected in the increase in the average settled claim at the different durations. This seems a proper and conservative basis of estimating. From studies of the 28 companies there appears to have been a tendency for early duration claims to have been overestimated whilst later duration claims have been underestimated. This is clearly a dangerous situation for increasing portfolios and one which the above method should bring out ${ }^{1}$ ).

30. It is, of course, not necessary to formally eliminate the effects of "mix" provided the changes concerned are identified and their continuance is reasonable, but care is necessary if estimates are made on the total portfolio. The claim pattern seems to break down into two major types (a) personal injury claims which have a long and distinctive settlement pattern and (b) damage claims which are largely disposed of within 2 years of incurment. (The differences between the two examples in para. 26 reflect this point). A subdivision into liability and own damage will not be an effective separation of the personal injury and third party damage claims and a change in the relative proportions of these two sections of cover will be reflected in a number of ways.

3I. For example an increase in the proportion of damage claims will increase the proportion of the total claims settled in the first year. A comparison of outstanding claims with settlements will then show an apparent fall. Since a similar feature would be shown if the "mix" had remained steady but the outstandings had been

1) See para. 6o for further comments on "inflation" 
underestimated, some care is required. If, of course, this feature had been a trend for a number of years, the various average settlements would reflect the movement and the resulting estimates would not be very much in error.

32. The trend of average claim settlements can be disguised by variations in the proportion of zero claims and this raises the question as to whether the basic final estimates should be made by estimating the proportion of non-nil claims from the total notifications and using an expected average non-nil claim, or whether the average claim used should be based on all claims, including nils. If the proportion of nil claims is steady there is clearly no difference in effect; if the proportion is changing due to a slow change in the composition of the business, the variation in the proportion of nil claims can be handled in the estimation of the increase in average claim. If the proportion of nils is subject to fluctuation, then it is probably preferable to estimate the number of non-nil claims (calculation of the ratio of non-nil claims to exposures provides a consistency check). In the data for the 28 companies all these methods were used at different times, it being remembered that the data were based on what could be conveniently provided rather than what would have been liked!

33. Another point which emerged from the study was the question of partial payments. The major part of these will arise when claims under different sections of a policy arise from a single accident and the accident is treated as one claim. The damage components will be settled fairly early and will then appear as part payments against the outstanding claims. If each section of the claim is treated separately then clearly the part payments will be relatively low. For a strict analysis the average settlement figures should be properly related to the numbers of claims settled. Sometimes however the information available may relate to paid claims, i.e. settlements plus partial payments on cases still outstanding. If the incidence of partial payments remains steady then the average paid claim, i.e. total payments divided by number of settled claims, will be a usable statistic, it being noted that the average will be higher than for settled claims, with, of course, a corresponding lowering of the average outstanding claim. 
34. If the "mix" should change however it may be difficult to sort out the true variation and the desirable method is to make sure that the settled amounts and numbers correspond. The data for the 28 companies showed a variety of situations.

35. On the general question of the degree of subdivision of the data a number of practical points emerged from the study. There are, of course, some differences in the average claim from the different types of cover, i.e. liability, both bodily injury and property damage and own damage. If the relative proportions change then the overall average will change merely from the change in mix. There need be no trouble in working with overall averages provided the change in mix is appreciated. For example, a portfolio consisting of two basic sizes of claims could show an overall constant average even though the separate averages were increasing if the change in proportions was appropriate. In making estimates of the future level of settlement provided due regard were given to the continuance of the changing mixture of business, no difficulty would arise. There are so many possible variations that it is difficult to lay down any hard and fast rule but the study suggested that extensive subdivision was not essential.

36. One particular aspect may be noted in connection with portfolios which consist of business with various amounts of excesses imposed. A study of this question showed that the major effect of an excess was to reduce the frequency, with only a minor change in the average value of the claim (average of cases involving some payment). In fact in the theoretical case in which the claim distribution is exponential it will be found that the average claim remains constant. This is, of course, a useful feature as it means that subdivision into excess classes is hardly necessary.

37. Another complication is the portfolio which consists of cases subject to no claims bonuses. The effect here is similar to an excess in that the frequency will fall sharply with higher bonus, but the average claim will show a much smaller variation. The separate consideration of frequency and average amount will mean that separation into bonus classes is hardly necessary for the purpose in view. 
38. Before turning to various checks which can be applied the following sets out the basic data required for the claims study:

\begin{tabular}{|c|c|c|c|c|}
\hline \multicolumn{5}{|c|}{ Year of Account 1965} \\
\hline $\begin{array}{l}\text { Year } \\
\text { of } \\
\text { notif1- } \\
\text { cation }\end{array}$ & $\begin{array}{l}\text { Amount } \\
\text { of claims } \\
\text { settled } \\
\text { in } 1965\end{array}$ & $\begin{array}{l}\text { No of No of Claim } \\
\text { claims zero s/ments } \\
\text { claims to } 1965\end{array}$ & $\begin{array}{l}\text { No of No of No o/s } \\
\text { claims zeros } 31 \text { I } 265\end{array}$ & $\begin{array}{l}\text { Provision } \\
\text { (1nc part } \\
\text { payments) }\end{array}$ \\
\hline $\begin{array}{l}1965 \\
1964 \\
1963 \\
1962\end{array}$ & & & & \\
\hline
\end{tabular}

Other internal checks

39. It is assumed that earned premiums are available so that for each year an average premium can be calculated from the exposed to risk. (If this latter time is not vehicle years but policy years there is no problem provided the porportion of fleet cases remains steady). Variations in this average will provide an indication of consistency in the premiums and exposures and also of tariff changes. The latter should, of course, be confirmed from other sources

40. The product of claim frequency rate and expected average settled claim will provide a measure of the risk premium which can be compared with the average earned premium adjusted appropriately for commission and expenses.

4I. Another check which can be made is to calculate the proportions of the claims settled at different durations. For the studies of the 28 companies the pattern showed a considerable stability for given types of claims in a given country. The figures can be found from the summary set out in para. 9 and comparisons made for successive years.

42. Following on the above comments calculation of the proportion of estimated total claims outstanding at the different durations also leads to a fairly steady pattern.

43. The average outstanding claim can also be calculated but study of the figures shows that the random variation of this statistic 
is fairly large and it becomes difficult to discern the true trend for the later durations. This brings out the point that the real comparison to be made is not the comparison of outstanding figures in each year of notification but the total figure. Tests of statistical significance are discussed later.

\section{Reinsurance}

44. The study of the 28 companies has been made on the basis of the gross figures, i.e. without adjustment for reinsurance premiums or claims recoveries. Most, if not all, motor accounts are protected by some form of excess of loss reinsurance, the effect of which is to relieve the primary insurer of the extreme tail of the claim distributions. However, since the premium for such reinsurances is usually linked closely to the actual excess claims, and terms will be rapidly adjusted if the experience deviates markedly from that expected, there is an arguable case for ignoring the reinsurance in making the basic calculations. If the excess level is low, so that a substantial proportion of the original premiums is passed to the reinsurer, then the position cannot be overlooked as provision has to be made for the expense and profit loadings imposed by the reinsurer. This can be done by appropriate adjustment to the expenses. The more difficult aspect is the appropriate fluctuation margin required and will be discussed later.

\section{Fluctuation Margin}

45. From supplemental figures giving the distribution of clajms by amounts some studies have been made on this subject and a few preliminary comments on the method of approach seem appropriate.

46. Provided the calculations of the expected values of claims on unexpired risks and on emerged claims have been appropriately made so as to eliminate any systematic variations the fluctuation margin can be determined from straightforward statistical theory. This theory leads to the distribution of amounts about the expected value and leads to quantities defined in the form of the probability that the actual value exceeds the expected value by a stated margin. 
47. There are two main components, i.e. the expected claims on the unexpired risks and on the emerged claims and these will first be considered separately.

\section{Unexpired Risks}

48. At the close of the accounting period it is assumed that an estimate is available of the unexpired exposure. From the calculations relating to the claim frequency rate an estimate can be made of the expected number of claims to arise in the future from these risks. For this purpose the frequency rate used should be an estimate of the experience to be expected and should thus be based on the trend of the values (para. 2I) and any information which is relevant to the problem. To the number so found should be added the expected number of late reported clajms and, as appropriate, the expected number of reopened claims.

49. These claims will be settled at average values appropriate to cases notified in the year following the date of closing the account and an appropriate average value found from the table in para. 9 . The product of those two items then gives the expected value of claims on the unexpired risks, the late reportings and reopened cases. However, the actual values at which these claims will be settled will be distributed round this expected value and the question is to determine the characteristics of this distribution. Formally the problem is one of "convolutions" but provided the number of expected claims is reasonably large, i.e. more than a few hundreds, calculation from the data provided by the 6 th Conference shows that a simpler method will be sufficiently accurate for the purpose in mind.

50. It has been shown (see e.g. ref. I) that the moments of the distribution of expected claims from a compounding of claims following a Poisson process with a claim distribution can be expressed as:

$$
\begin{gathered}
m_{1}^{p}=N p m_{1}, \mu_{2}^{p}=N p m_{2}, \mu_{3}^{p}=N p m_{3}, \\
\mu_{4}^{p}=N p m_{4}+3 N^{2} p^{2} m_{2}^{2}
\end{gathered}
$$

where $N$ is the number of risks exposed, $p$ the expected claim frequency rate and $m_{n}$ are the moments of the claim distribution. The Pearson moment functions $\beta_{1}$ and $\beta_{2}$ for this distribution are 
$\frac{\mathrm{I}}{N p}\left(m_{3}^{2} / m_{2}^{3}\right)$ and $3+\frac{\mathrm{I}}{N p}\left(m_{4} / m_{2}^{2}\right)$ respectively. Calculations relating to actual claims distributions show that the expressions in brackets are of the order of a few hundred, but generally considerably less, so that provided $N p$ is of the order of, say, I,000, the distribution of total amount of claims will be fairly closely approximated by the normal curve for deviations not too remote from the mean. For smaller values of $N p$ the normal curve would provide too low an estimate of the probability, but the tables in ref. 2 are sufficient to enable an adjustment to be easily made.

5 I. The problem thus reduces to estimating the standard deviation of the total claim distribution. If a distribution of settled claims is available $m_{2}$, the second moment about zero, can be calculated. This will not be precisely the quantity required since we are concerned with notified claims and their future settlement pattern. (The settled claim distribution will reflect the past growth of business because of the time lag in settlement of the larger claims). However calculations have been made for a number of claim distributions and the coefficient of variation $\sigma / m_{1}$ found. These calculations show that for own damage claims the coefficient is of the order of $\mathrm{x} .8$, for liability claims and for mixed liability and damage claims values of from 2.5 to 5.0 appear, with a general tendency to cluster round about 3.0. For "mixed" portfolios a figure of about 3.5 was found to be representative. Each country tested showed distinctive values so that it is not possible to generalise on the value to be used but calculations must be made to establish the appropriate figure. When this is known the standard deviation can be simply expressed as a multiple of the mean value.

52. Some adjustment should be made to allow for the fact that the claim process is generally speaking not a simple Poisson process. From other data an increase of $30 \%$ in the standard deviation would not be an unreasonable adjustment. This figure of $30 \%$ is appropriate for values of $n$ of the order of a few thousand, but a more accurate formula, although still appropriate, would be a multiplier of $\sqrt{\mathrm{r}+n / 5000}$ instead of $\mathrm{r} .30$.

53. If, for example, the coefficient of variation for a particular country was considered to be 3.5 , the standard deviation of the 
mean total claim $(m)$ would be taken as $3.5 \times \mathbf{I} .3 \times m$, or, say, $5^{m}$ to allow a margin for non-normality. If the expected number of claims is $n$ then we have:

$$
\begin{aligned}
\text { Total expected claims } & =n m \\
\text { approx. S.D. of " }, & =5 m \sqrt{n}
\end{aligned}
$$

and we could say with some degree of confidence that the probability that the total claims would exceed $m n+15 m l n$ is only about $I$ in $I, 000$.

\section{Outstanding Claims}

54. The situation with the outstanding claims is slightly different as we are concerned with the variation in the settlement values of the actual number of emerged claims, i.e. apart from the complication of zero claims, the sample size is fixed and not subject to random variation as in the case of unexpired risks. Suitable statistics are available from one or two companies from which to calculate the coefficient of variation for the settled claims at different durations for a particular year's incurred claims and this was found to be lower at all durations than the figure for the full year's incurred claims. An estimate based on these figures for the coefficient of variation for the total outstanding claims gave a figure slightly above that found for the incurred claims. This suggests, since the $\beta_{1}$ and $\beta_{2}$ values were if anything rather smaller than for the outstanding claims, that the standard deviation of outstanding claims can be found by using a similar multiplier. The estimated outstanding amounts are given from the table in para. 9 and the numbers from the table in para. 2I. Hence we have:

$$
\begin{aligned}
\text { Total outstanding claims } & =n_{0} \times m_{0} \\
\text { approx. S.D. of , ", } & =5 m_{0} \sqrt{n_{0}}
\end{aligned}
$$

55. We may calculate the standard deviation of the total of expected claims on the unexpired risks and on the outstanding claims as approximately

$$
5 \sqrt{m^{2} n+m_{0}^{2} n_{0}}
$$

which will not differ greatly from $5 \bar{m} \sqrt{n+n_{0}}$, where $\bar{m}$ is derived from the total amounts of the two claim items. 
56. This now leads to a simple formula for finding an approximate value for the fluctuation margin. For the company discussed above we determine the quantities-:

Numbers Amounts

(a) Expected claims on unexpired risks $n \quad m \times n$

(b) Outstanding claims $n_{0} \quad m_{0} \times n_{0}$

Total $n_{t} \quad \bar{m} \times n_{t}$

The fluctuation margin at the I per mille level is then approximately

$$
\text { I5 } \bar{m} \sqrt{n_{t}}
$$

57. It will be noted that the fluctuation reserve increases in proportion to the square root of the total number of claims (unexpired risks and outstanding). For a mature company this latter number will be roughly proportional to the premium income so that the theoretical reserve is roughly proportional to the square root of the premium income, confirmation that a formula based on a constant multiplier related to income will not be satisfactory. Either it will give reasonable values for small incomes, in which case it will be too high for large incomes, or if it is right for large incomes it will be too small for low income companies.

58. It may also be noted that the fluctuation reserve derived above is not dissimilar in form to the risk reserve derived from risk theoretical studies. For example, Pentikainen has shown that the reserve required is of the form

$$
k \sqrt{P}-\lambda P
$$

where $k$ is a parameter dependent on the risk structure of the company and $\lambda$ is the profit margin in the premiums $P$. If the profit is ignored, then the risk reserves and the above fluctuation reserve are identical in form. If $\lambda$ is positive, i.e. the business is profitable, then the risk reserve required is lower by the equivalent of a year's expected profit. If $\lambda$ is negative then an increase is required equivalent to a year's expected loss.

59. It thus appears that for motor business the calculation of a fluctuation reserve need not involve any very elaborate computations. There is little justification from the nature of the problem for a precise calculation of the distribution of total claims. In the 
first place the estimates of expected values involve a number of quantities which cannot be fixed with great precision and in the second place the estimates of the parameters of the claim distribution are themselves subject to substantial sampling errors. This does not mean that the multipliers for the standard deviations should not be selected with care. They differ between classes of business and must be fixed with some regard to the make up of the portfolio, but the technique of para. 55 is adequate to deal with practical situations.

\section{Inflation}

6o. A few words of comment are pertinent on the subject of the increase in average claims with time. There appear to be two main components covering the variation other than changes in policy covers. The first arises from the fact that the larger proportion of motor claims are linked to the level of wages, either from the evaluation of third party injury awards or from the labour cost involved in repairs. The level of wages may change from an increase in real wealth or from a decrease in the value of money but past statistics relating to the growth in claims costs will not distinguish between the two effects. The second component arises from the tendency for claims incidence to increase from a greater appreciation that recovery is possible and from a tendency for awards to become relatively more generous.

6r. In studying past statistics of settled claims the trends will reflect, inter alia, both of these factors and in using the past to estimate the future due regard must be had to the causes of the trends. Unless there has been some special change in the legal basis causing an increase in awards, it is not unreasonable to assume the continuation of recent past trends in the basis of court awards and extrapolation is justified. So far as changes in monetary values are concerned it also seems reasonable to allow for a continuation of change arising from greater prosperity, i.e. increase in real wage levels. The position regarding purely inflationary increases is however not so clear. It would seem questionable, other than as a safety measure, to automatically assume that future inflation will continue as in the past in which case estimates of future settlements should be based on a rather slower rate of 
increase than shown from past trends. The question of how much slower is not easy to answer and can only be determined against the background of conditions in a particular country.

62. To try and provide an answer it seems necessary to take a broader look at the problem. In the foregoing calculation no allowance has been made in the calculations for the time element in the distribution of claims. In reality, interest will be earned on the claims reserves so that provided it was understood that interest so earned was needed for claims purposes, it would be possible to determine a discounted value for the expected future claims payments. If there was a degree of inflation, this might well be reflected in the rate of interest and the higher estimates based on inflation could be discounted at a higher rate of interest. However, the claims payments form a fairly rapidly reducing series and it would seem desirable to so invest the claim reserve that capital depreciation was minimised. With this condition the setting off of extra interest earnings against inflation of claim values would seem to be of limited application only.

63. We are thus left with the situation that so far as inflationary increases in claims are concerned a decision must be made either to make some estimate for future inflation based on the past trends or to deliberately ignore inflation in the estimating. In the first case absence of inflation will mean that there has been some degree of overprovision; in the latter case there will be underprovision if there is inflation. The dilemma is a real one for supervisory officials as the amount involved is not unimportant. A rough calculation based on a fairly long liability "tail" suggests that $5 \%$ inflation a year would mean something like a $10 \%$ increase in the cost of outstanding liability claims.

64. In all the circumstances the simplest basis seems to be to base the estimates of future settlements on the trends derived from the settled claims and to consider the degree of inflation included in these past records. If this is small, and the economy of the country suggests that it will remain so, the estimates need no adjustment. If there has been a marked recent inflation, which has been brought under control, some adjustment could be made in the future estimates of average claims. No provision can be made 
for unforeseen sudden inflation for which the only defence seems to be contingency reserves as distinct from the claim fluctuation reserves which are specifically calculated to cover random fluctuations. It will be appreciated that these considerations relating to inflation are inherent in any method of estimating future claims outgo, but the use of average expected settlements does provide a convenient method for making any adjustments considered necessary.

\section{Rapidly growing accounts}

65. The estimation of reserves required for a rapidly growing account is an important question, particularly for motor liability business. It is convenient to distinguish between an established company which is growing fast and a new company which will be discussed later.

66. The evidence from the study of the 28 companies was that the basic principle of constant claim frequency rate did hold so that the first requirement is a calculation of the claim frequency rate. This depends on the exposure to risk and the numbers of claims; various methods of finding a sufficiently accurate exposure can be devised but some care is needed when the account is growing fast, particular attention being given to avoid overestimation. The possible sources of errors in the reporting of claims have been mentioned earlier and it is sufficient to mention that the incidence of late reportings and reopened cases may be relatively heavy so that particular attention must be given to these in studying the company returns.

67. As regards the average claim it would seem that the calculations of Ist year settled averages, Ist and 2nd year averages etc. will reveal the pattern of increase in average claim appropriate to the company. There may be insufficient data from the company figures to provide a confident estimate of the ultimate settled average but comparison with corresponding figures for other companies should show a reasonable relationship between the average first year settled claims in the different companies, due regard being had to any significant differences in composition, 
and enable a reasonable estimate to be made of the likely ultimate settled figure.

68. The case of a new company had a great deal in common with a rapidly growing company except that the amount of past data will be small-in fact there may be no reliable settled claims data at durations after the first year. In the circumstances the average first year settlement may be the only reliable statistic but provided this is comparable with corresponding figures from other companies there need be no hesitation in developing expected settled incurred average figures. In other words if the average first year settled claim is $£ 5^{\circ}$ and the corresponding figure for another company with a roughly similar type of business is $£ 5^{2}$, rising to an average of $£ 80$ when all the claims are settled, it would be reasonable to use a figure of $£ 80$ as the expected incurred average claim. If the company estimate (i.e. paid plus outstanding) for the year in question was appreciably below this figure there would be prima facie evidence of underprovision and further investigation called for. It will be appreciated that in these sort of conditions the company may well have no developed claims experience at the longer durations as most of the larger claims may take at least two or three years before settlement or a reliable estimate can be formulated.

\section{Concluding comment}

69. The analysis made of the 28 companies' data confirms the suggestion that provided statistics are properly and consistently compiled, subdivision into claim frequency and average claim does provide a practical method of estimating reserve requirements. It has not yet been practicable to examine the data to see if alternative methods are as effective or more effective. The limitations of the data as regards extension in time and also having regard to the fact that the statistics were to some extent those available rather than those derived have necessarily restricted the analysis.

\section{REFERENCES}

[I] R. E. BEARD: Jnl. Inst. Act. Students Society - Vol. I3, p. I39 (1955).

[2] N. L. Johnson, E. Nixon, D. F. Amos: Biometrika - Vol. 50, p. 459 (1963). 